\title{
Kleine-Levin syndrome and bipolar disorder: a differential diagnosis of recurrent and resistant depression
}

Pierre Alexis GEOFFROY ${ }^{1,3,4,5^{*}}$, Isabelle ARNULF ${ }^{6}$, Bruno ETAIN ${ }^{1,2,3,5}$, Chantal HENRY ${ }^{1,2,3,5}$.

1) INSERM, U955, Créteil, 94000, France ;

2) Université Paris Est, Faculté de médecine, Créteil, 94000, France ;

3) AP-HP, Hôpital H. Mondor - A. Chenevier, Pôle de Psychiatrie, Créteil, 94000, France ;

4) Pôle de psychiatrie, Univ Lille Nord de France, CHRU de Lille, F-59000 Lille, France ;

5) Fondation FondaMental, Créteil, 94000, France.

6) Unité des pathologies du sommeil, centre de référence narcolepsie, hypersomnie et syndrome de Kleine-Levin, Inserm U975 et CRICM Hôpital Pitié-Salpêtrière, Paris

\section{*Correspondence should be sent to:}

Pierre Alexis GEOFFROY, MD

Pôle de Psychiatrie, Centre Expert Bipolaire

Hôpital Albert Chenevier, pavillon Hartman

40, rue de Mesly

94000 Créteil Cedex - FRANCE

Tel: + 33149813290 - Fax: + 33149813099

E-mail: pierre.a.geoffroy@gmail.com

Conflict of interest: Authors declare having no actual or potential conflict of interest that could influence, or be perceived to influence, this work.

Funding sources: none

Words: 1551 /2000 (abstract: 250 /250)

\section{Tables: 1}

\section{References: 8}




\section{ABSTRACT}

Objectives: Kleine-Levin syndrome (KLS) is a rare sleep disorder (1-2 cases per million inhabitants) primarily affecting young subjects. Episodes are characterized by hypersomnia, cognitive impairment, feelings of derealization, and less frequently hyperphagia (66\%), hypersexuality (53\%, principally men) and depressed mood (53\%, predominantly women). KLS cases are frequently misdiagnosed and pose therapeutic challenges: amantadine and mood stabilizers are only marginally effective. The cause of KLS is unknown, but an association between KLS and mood disorders is suggested by some clinical analogies.

Methods: We report the case of a 44-year-old Caucasian woman with bipolar disorder (BD) subtype I referred by her psychiatrist to our bipolar expert center for recurrent and resistant major depression according to DSM-IV TR criteria.

Results: We confirmed the BD I and KLS diagnoses. About 15 evenly distributed KLS episodes, each lasting about a week, occurred during the 6 years following the first manifestation at age 18 years. An electroencephalogram was performed during a KLS episode and showed high amplitude theta waves in left and right temporal lobes, predominant in the left hemisphere. The pronounced BD symptomatology -starting at 15 years-old with major depression and suicide attempt - had impeded the identification of KLS, which was not diagnosed until age 42 years.

Conclusions: BD may obscure KLS with each condition adversely affecting the course of the other. Consequently, the co-occurrence of KLS and BD may be underestimated. KLS and BD may share common vulnerability factors, such as immune-inflammatory and circadian disturbances, and there may be a genetic predisposition for both. Mood stabilizers may be effective for KLS and BD. 
KEYWORDS: Kleine-Levin Syndrome, Bipolar Disorder, Hypersexuality, Hyperphagia, Hypersomnia.

\section{Introduction}

Kleine-Levin syndrome (KLS) is a rare sleep disorder (1-2 cases per million inhabitants) primarily affecting young subjects (1). The cause of KLS is unknown, although nearly half of such patients experience a common flu-like infectious disease a few days before the onset of their first KLS episode. This is suggestive of an autoimmune mechanism and genetic predisposition (1). Episodes are characterized by hypersomnia (15-21 hours per day), cognitive impairment (apathy, confusion, slowness, amnesia), specific feelings of derealization (dreamy state, altered perception), and less frequently hyperphagia (66\%), hypersexuality (53\%, principally men) and depressed mood (53\%, predominantly women) (1). KLS cases are frequently misdiagnosed and pose therapeutic challenges: amantadine and mood stabilizers are only poorly effective (1). An association between KLS and mood disorders has been suggested on the basis of various clinical analogies and because of shared possible pathogenic mechanisms, such as disruption of circadian rhythms (2). We found only two case-reports of recurrent hypersomnia associated with manic-depressive episodes in the literature $(3,4)$.

We report here a case of KLS associated with bipolar disorder (BD). The association of these two diseases leads to issues of differential diagnosis with recurrence of depression and problems in the management of treatment. 


\section{Methods}

We report the case of a 44-year-old Caucasian woman with BD subtype I referred by her psychiatrist to our bipolar expert center for recurrent and resistant major depression and already diagnosed as BD according to DSM-IV TR criteria (5).

\section{Results}

The patient presented with a long medical history starting without specific abnormality: a birth after prolonged labor without any particular difficulty, satisfactory psychomotor development and puberty at age 12-years. She has two cousins with schizophrenia in her maternal family. Her mood history includes a first suicide attempt at 8 years-old with "cooking gas" followed by a suicide attempt by massive intake of drugs during a major depressive episode when 15 years-old. BD was diagnosed at age 30 years following a manic episode with psychotic features in post-partum. Thereafter, she received specialized psychiatric follow-up. Her bipolar history (summarized in table 1) started at age 15 years and was marked by several depressive, hypomanic and three manic episodes with psychotic features (grandiose and mystic delusions without hallucinations). At the time of assessment, she was unable to work or to take care of her son, and stayed at home day and night with a reduced activity (watching TV and smoking in a dark kitchen), totally supported by her mother or from wider society.

She was referred to our expert center for $\mathrm{BD}$ and recurrent and resistant major depressions. We confirmed the BD I diagnosis and carefully reviewed her medical history. In addition to mood episodes, the patient described periods that she called "coma". A careful interview with the patient revealed hypersomnia episodes starting at age 18 years and that were entirely distinct from her affective disorder: the patient suffered from intermittent episodes with hypersomnia, loss of sleep rhythms, disturbances of awareness with confusion 
and apathy, depressive symptoms, feelings of derealization and of imminent death, behavioral abnormalities, and hyperphagia with complete amnesia about the occurrence of these episodes 26 years earlier. Her mother, who described very accurately the symptomatology, confirmed these episodes. The symptomatology was entirely consistent with KLS. About 15 evenly distributed episodes, each lasting about a week, occurred during the first 6 years after the first manifestation. Subsequently, the frequency decreased to 1-3 episodes annually, the duration shortened to a few days, and the severity of symptoms decreased gradually. When she was 19 years old, the patient underwent a brain scan (which was normal). An electroencephalogram (EEG) was performed during a KLS episode and showed high amplitude theta waves in left and right temporal lobes, predominant in the left hemisphere, which reflected the EEG abnormalities associated with KLS (6). Unfortunately, the condition had not been diagnosed. At time of assessment, the patient had been free of clear KLS episodes for 20 years. However, soon after repetitive transcranial magnetic stimulation (rTMS) treatment for resistant depression, she reported a major, continuous apathy for 2 years, with variable sleep times (518 hours' subjective sleep/day), but no derealization or cognitive problems. The KLS history is summarized in Table 1.

To confirm the diagnosis of KLS and to obtain advice for the current persistent sleep disturbances, the patient was referred to a sleep and KLS referral center. Her physical examination, brain scan, computed tomography brain imaging, metabolic parameters, antibody panel, tests for parasites (including trypanosomiasis family), and cerebrospinal fluid analysis, were within normal limits and excluded other neurological and systemic causes that could explain the recurrent hypersomnic symptoms. In particular, the brain scintigraphy, which is abnormal in $75 \%$ patients with KLS between episodes, and consistently abnormal during episodes, was normal. A neuropsychological test battery was performed that did not reveal any abnormalities except a minor attention deficit with good memory performance. 
Actigraphy (movement recorded over extended periods by a portable device to study sleep and circadian rhythms) was used to explore the periods of "hypersomnia": the analysis of a recent phase of "hypersomnia" showed a slightly increased (>10 h) sleep time, followed by days with less than $5 \mathrm{~h}$ of sleep, and a fragmented, irregular sleep-wake rhythm, conforming to a pattern typical of mood disorders. None of these abnormalities were consistent with a prolonged typical KLS episode.

The patient did not fulfill the full DSM-IV criteria for major depression but suffered from apathy and hypersomnia. She has been treated for her BD intermittently with various antipsychotics, antiepileptics, and mood stabilizers. The treatment with mood stabilizers, despite being recognized in KLS, had started only after her first manic episode because KLS was not diagnosed at that time. Recently, she had been receiving 1000mg/day lithium associated most of the time with antidepressants; this treatment failed to improve these recurrent episodes. For several years she had been treated with the antidepressant Amineptine (Survector), a selective dopamine reuptake blocker with psychostimulant effects. Amineptine was removed from the market by the medication regulatory agency due to its addictive risks. At that time, the patient was able to work and there was no recurrence of hypersomnia. In an attempt to treat her depression by bypassing her resistance to antidepressants, rTMS treatment was initiated two years later but provided no benefit. Finally, following the diagnosis of KLS, Modafinil was gradually added (100 to $300 \mathrm{mg}$ in the morning) as an adjuvant to treat the perceived apathy: the patient declared that this treatment was beneficial during the first two months, but had then led to a shift to a sub-manic state and been stopped. Most recently, we re-introduced modafinil at a lower dose, with a strict mood monitoring, as the best option available. 


\section{IV.Discussion}

This description of KLS associated with BD is surprising considering its complex presentation with an overlap between the clinical symptoms of the two syndromes. KLS was only diagnosed one year ago, at age 42 years, despite the first episode being at 18 years old. The pronounced BD symptomatology undoubtedly impeded the identification of KLS. Sleep disturbances were considered to be part of an atypical profile of depression with recurrence and resistance to treatment. In contrast with $\mathrm{BD}$, the diagnosis of which was not difficult in this case, KLS patients generally do not report psychiatric symptoms between episodes and have no significant family history of psychiatric disorders (1). A recent review of KLS subjects highlighted that it is common for patients to be misdiagnosed with a psychiatric entity and even in some cases hospitalized, with negative consequences due to the exacerbation of the cognitive symptoms in novel environments (1). Here, we report a patient with misdiagnosis of KLS because of a true BD diagnosis, with psychiatric family history; this misdiagnosis had negative social and professional consequences because the KLS remained untreated. We cannot determine whether or not there is a link between the two diseases in this patient, but BD clearly preceded the first KLS episode. BD was also of very severe in this patient, with resistance to most antidepressants and an evolution toward an apathetic status. KLS is a brain disease with evidence of temporal and frontal lobes hypoperfusion frequently persisting after the onset of episodes, and it is possible that the KLS had made this patient more vulnerable to BD and increased the severity of the psychiatric disease. Whether KLS is a risk factor for long-term psychiatric diseases remains to be determined: large, prospective series would be required.

We agree with Arnulf et al. (1) who suggest that KLS patients are frequently misdiagnosed as suffering from psychiatric diseases. We also believe that the co-occurrence of KLS and BD may be underestimated in clinical practice because the heterogeneity of the 
symptomatology of BD (like atypical depression symptoms) may cloud the identification of KLS. Care and prognosis for BD patients may be adversely affected if KLS is missed, as in this case.

Another important point raised by this case is the possible shared vulnerability to the two diseases. Current hypotheses for both diseases include immuno-inflammatory vulnerability with genetic predisposition $(1,7)$. Moreover, disruption of circadian rhythms has been suggested in KLS (1) and is well documented in BD (8).

In conclusion, BD may impede identification of KLS and thereby adversely affect the course of both diseases. KLS patients are often misdiagnosed as suffering from psychiatric diseases, and this case illustrates how the co-occurrence of KLS and BD may be underestimated in clinical practice. KLS and BD may share vulnerability factors, particularly immune-inflammatory and circadian disturbances with a genetic predisposition for both. However, psychiatric disorders are not frequently reported in KLS familial history. Mood stabilizers may show efficacy for KLS and BD. However, KLS needs specific management: primarily supportive and educational, and secondarily chemical with lithium, valproate or amantadine (1). 


\section{$\underline{\text { References: }}$}

1. Arnulf I, Lin L, Gadoth N, et al. Kleine-Levin syndrome: a systematic study of 108 patients. Ann. Neurol. 2008; 63(4):482-93.

2. Billiard M, Jaussent I, Dauvilliers Y, et al. Recurrent hypersomnia: a review of 339 cases. Sleep Med Rev. 2011; 15(4):247-57.

3. Wilder J. A case of atypical Kleine-Levin syndrome: 30 years' observation. J. Nerv. Ment. Dis. 1972; 154(1):69-72.

4. Jeffries JJ, Lefebvre A. Depression and mania associated with Kleine-Levin-Critchley syndrome. Can Psychiatr Assoc J. 1973; 18(5):439-44.

5. Association AP. Diagnostic and Statistical Manual of Mental Disorders DSM-IV-TR Fourth Edition. 4th ed. Arlington: American Psychiatric Publishing, Inc.; 2000.

6. Arnulf I, Zeitzer JM, File J, et al. Kleine-Levin Syndrome: A Systematic Review of 186 Cases in the Literature. Brain. 2005; 128(12):2763-76.

7. Hamdani N, Tamouza R, Leboyer M. Immuno- inflammatory markers of bipolar disorder: a review of evidence. Front Biosci (Elite Ed). 2012; 4:2170-82.

8. Etain B, Milhiet V, Bellivier F, et al. Genetics of circadian rhythms and mood spectrum disorders. Eur Neuropsychopharmacol. 2011; 21 Suppl 4:S676-682. 
Table 1:

Summary of the patient's medical history

\begin{tabular}{|c|c|c|}
\hline $\begin{array}{c}\text { Bipolar Disorder } \\
\text { history }\end{array}$ & $\begin{array}{c}\text { Age } \\
\text { (years) }\end{array}$ & $\begin{array}{l}\text { Kleine Levin Syndrome (KLS) } \\
\text { history }\end{array}$ \\
\hline $\begin{array}{l}\text { First suicide attempt } \\
\text { (with cooking gas) }\end{array}$ & 8 & \\
\hline $\begin{array}{l}\text { Second suicide attempt } \\
\text { (with drugs) } \\
+ \text { Major depression }\end{array}$ & 15 & \\
\hline $\begin{array}{c}\text { Several depressive episodes } \\
\text { mood stabilizers having poor or no } \\
\text { efficacy }\end{array}$ & 15 to 44 & \\
\hline \multirow[t]{3}{*}{$\begin{array}{c}\text { Several hypomanic episodes } \\
\text { (uncounted) }\end{array}$} & 15 to 44 & \\
\hline & 18 & $\begin{array}{c}\text { First KLS episode } \\
\text { (diurnal EEGs showed high-amplitude } \\
\text { theta waves in the bilateral temporal lobes) }\end{array}$ \\
\hline & 18 to 23 & $\begin{array}{c}15 \text { KLS episodes } \\
\text { (each lasting about a week) }\end{array}$ \\
\hline $\begin{array}{l}\text { Third suicide attempt } \\
\text { (with drugs) }\end{array}$ & 21 & \\
\hline \multirow[t]{2}{*}{$\begin{array}{l}\text { Fourth and fifth suicide attempts } \\
\text { (with drugs) }\end{array}$} & 23 & \\
\hline & $23-44$ & $\begin{array}{l}\text { Several minor recurrent hypersomnia } \\
\text { episodes with apathy sequelae }\end{array}$ \\
\hline $\begin{array}{l}\text { First Mania with psychotic } \\
\text { symptoms in post-partum }\end{array}$ & 30 & \\
\hline $\begin{array}{l}\text { Second mania with psychotic } \\
\text { symptoms }\end{array}$ & 32 & \\
\hline \multirow[t]{2}{*}{$\begin{array}{l}\text { Third and last mania with psychotic } \\
\text { symptoms }\end{array}$} & 36 & \\
\hline & 40 & rTMS $^{*}$ treatment without benefit \\
\hline Euthymic bipolar disorder & $\begin{array}{c}44 \\
\text { (Today) }\end{array}$ & Continuous apathy \\
\hline
\end{tabular}

*rTMS = repetitive transcranial magnetic stimulation, initiated for a depression resistant to drug treatment. 